\title{
Diagnostic Reliability and Validity of the Semi- Structured Assessment for Drug Dependence and Alcoholism (SSADDA) Chinese Version
}

\author{
Bao-Zhu Yang a,b Liang-Jen Wang ${ }^{c}$ Ming-Chyi Huang ${ }^{d, e}$ Sheng-Chang Wang ${ }^{f}$ \\ Meng-Chang Tsai ${ }^{9} \quad$ Yu-Chi Huang ${ }^{\text {Yaira Z. Nuñez }}{ }^{a, b} \quad$ Mei-Hing Ng ${ }^{\text {h }}$ \\ Henry R. Kranzler ${ }^{i, j}$ Joel Gelernter ${ }^{a, b, k}$ Chih-Ken Chen ${ }^{1, m}$ \\ aDepartment of Psychiatry, Yale University School of Medicine, New Haven, CT, USA; bVA Connecticut Healthcare \\ System, West Haven, CT, USA; 'Department of Child and Adolescent Psychiatry, Kaohsiung Chang Gung Memorial \\ Hospital and Chang Gung University College of Medicine, Kaohsiung, Taiwan; 'Department of Addiction Sciences, \\ Taipei City Psychiatric Center, Taipei City Hospital, Taipei, Taiwan; 'Department of Psychiatry, School of Medicine, \\ College of Medicine, Taipei Medical University, Taipei, Taiwan; 'Center for Neuropsychiatric Research, National \\ Health Research Institutes, Miaoli, Taiwan; ${ }^{9}$ Department of Psychiatry, Kaohsiung Chang Gung Memorial Hospital, \\ and Chang Gung University College of Medicine, Kaohsiung, Taiwan; ${ }^{\text {h}}$ Department of Addiction, Tsaotun Psychiatric \\ Center, Nantou, Taiwan; 'Department of Psychiatry, University of Pennsylvania Perelman School of Medicine, \\ Philadelphia, PA, USA; 'VISN 4 Mental Illness Research, Education and Clinical Center, Crescenz VAMC, Philadelphia, \\ PA, USA; ${ }^{\text {Y }}$ ale University School of Medicine, Departments of Genetics and Neuroscience, New Haven, CT, USA; \\ 'Department of Psychiatry \& Community Medicine Research Center, Chang Gung Memorial Hospital, Keelung, \\ Taiwan; ${ }^{\mathrm{m} C h a n g}$ Gung University School of Medicine, Taoyuan, Taiwan
}

\section{Keywords}

Chinese Semi-Structured Assessment for Drug Dependence and Alcoholism - Diagnostic instrument - Ketamine dependence $\cdot$ Semi-structured interview $\cdot$ Test-retest reliability

\section{Abstract \\ The Semi-Structured Assessment for Drug Dependence and Alcoholism (SSADDA) is a polydiagnostic instrument for sub- stance use and psychiatric disorders. We translated the SSADDA English version into Chinese (SSADDA-Chinese) and report here our examination of the diagnostic reliability and validity of DSM-IV substance dependence (SD) diagno- ses in a Mandarin-speaking sample in Taiwan. We recruited 125 subjects who underwent an assessment of lifetime SD}

karger@karger.com www.karger.com/cxp

Karger $\stackrel{\text { ' }}{5}$

GOPEN ACCESS
(C) 2020 The Author(s)

Published by S. Karger AG, Basel

This is an Open Access article licensed under the Creative Commons Attribution-NonCommercial-4.0 International License (CC BY-NC) (http://www.karger.com/Services/OpenAccessLicense), applicable to the online version of the article only. Usage and distribution for commercial purposes requires written permission. diagnoses using both the SSADDA-Chinese and the Structured Clinical Interview for DSM-IV, Clinician Version (SCIDChinese). Thirty-one subjects were retested with the SSADDA-Chinese. Cohen's K statistic, which measures chance-corrected agreement, was used to measure the test-retest reliability and concurrent validity of the individual SD diagnoses. There was a high degree of concordance between SD diagnoses made using the SSADDA-Chinese and the SCIDChinese, including those for dependence on alcohol ( $\mathrm{K}=$ $0.83)$, ketamine $(\mathrm{K}=0.97)$, methamphetamine $(\mathrm{K}=0.93)$, and opioids $(k=0.95)$. The test-retest reliability of dependence diagnoses for ketamine $(\mathrm{K}=0.95)$, methamphetamine $(\mathrm{K}=$ 0.80 ), and opioids ( $k=1.00$ ) obtained using the SSADDA-

Bao-Zhu Yang and Liang-Jen Wang contributed equally.
Chih-Ken Chen

Department of Psychiatry, Chang Gung Memorial Hospital

No. 200 Lane 208, Jijin 1st Rd.

Keelung City 204 (Taiwan)

kenchen@cgmh.org.tw 
Chinese was excellent, while that for alcohol dependence $(\mathrm{K}=0.63)$ and nicotine dependence $(\mathrm{K}=0.65)$ was good. We conclude that the SSADDA-Chinese is a reliable and valid instrument for the diagnosis of major SD traits in Mandarinspeaking populations.

(C) 2020 The Author(s)

Published by S. Karger AG, Basel

\section{Introduction}

Substance misuse is a serious social and public health problem worldwide [1]. An estimated 25 million people, about $5 \%$ of the global adult population, used drugs at least once in 2015 [1]. Further, illicit drug use and dependence contribute significantly to the global burden of disease and directly to 20.0 million disability-adjusted life years in 2010 [2]. Thus, the development of reliable and valid instruments to assess the diagnosis of substance use disorders (SUDs) in languages other than English is imperative both for research purposes and clinical practice in different regions of the world.

The Semi-Structured Assessment for Drug Dependence and Alcoholism (SSADDA) is a comprehensive interview first developed in English for use in genetic studies of substance dependence (SD) and related psychiatric disorders $[3,4]$. It was based on the Semi-Structured Assessment for the Genetics of Alcoholism (SSAGA) [5, 6], which was designed for use in genetic studies of alcohol dependence. The SSADDA provides broad coverage of various SD or SUD traits as well as other major psychiatric diagnoses based upon DSM-IV [3] or DSM-5 criteria of diagnoses [7], respectively. The SSADDA, similar to the SSAGA, includes detailed questions on the onset and recency of criteria for individual substances and evaluates the criteria's temporal clustering [4]. In contrast to the SSAGA, the SSADDA also includes sections on attention deficit hyperactivity disorder, gambling disorder, and childhood experiences [4], which have been shown to affect the risk of alcohol and drug use $[8,9]$.

The test-retest reliability of diagnoses made using the SSADDA was previously shown to be excellent for DSMIV nicotine dependence and opioid dependence (OD), good for alcohol dependence and cocaine dependence, and fair for cannabis dependence, sedative dependence, and stimulant dependence $[3,4]$. The interrater reliability was good to excellent for most of the common SD diagnoses; for both OD $(\kappa=0.91)$ and cocaine dependence $(\kappa=0.83)$, the interrater reliability was excellent [3].

The SSADDA has been translated into a Thai version [10], for which the interrater reliability and concurrent

Reliability and Validity of the Chinese SSADDA validity of DSM-IV OD have also been evaluated [10]. The Thai SSADDA showed excellent interrater reliability for OD $(\kappa=0.97)$ and excellent concurrent validity when compared to the Thai MINI-Neuropsychiatric Interview $(\kappa=1.00)$. In addition, the SSADDA alcohol dependence section was translated into Samoan and tested in ethnic Samoans. It was found to have good-to-excellent overall test-retest reliability of both DSM-III-R and DSM-IV alcohol dependence diagnoses, though the reliability was greater when it was administered in English than in either Samoan or both languages [11].

In recent years, substance misuse has been linked to many social issues and serious public health problems in Taiwan [12] and China [13]. Mandarin Chinese is spoken most often in Taiwan and China [14]. Given the large Mandarin-speaking populations there, to assess substance misuse by means of the SSADDA in Taiwan or China, the SSADDA would be in great need of a Chinese version with validation. To accomplish this, we translated the SSADDA into Chinese and adapted it to the drug use habits of the Chinese population by developing a new section on ketamine use and replacing the cocaine section with a methamphetamine section. In Taiwan, in 2014, the most widely used drug was methamphetamine $(0.60 \%)$, followed by ketamine $(0.39 \%)$, marijuana $(0.30 \%)$, heroin $(0.23 \%)$, and ecstasy (0.20\%) [12].

Although the SSADDA can be used to assess DSM-5 diagnoses [7], we chose to use the DSM-IV diagnoses in this study to allow us to compare our findings with those from 3 previous psychometric studies of the SSADDA [3, $4,10]$. These include 2 published reports on the reliability of the English version of the SSADDA and 1 published study on the Thai version of the SSADDA, which were based on DSM-IV diagnostic criteria. In terms of the diagnostic criteria for SUDs, DSM-IV and DSM-5 are similar. The major difference in criteria is that the legal problems criterion from DSM-IV was replaced in DSM-5 by craving (i.e., a strong desire or urge to use a substance). A major difference between the systems is that DSM-IV includes both substance abuse and SD, while DSM- 5 has 3 categories of severity of SUD and no distinction between abuse and dependence; that is, $2-3$ criteria are diagnosed as mild, $4-5$ criteria as moderate, and 6 or more criteria as severe SUD. Further, the physiological subtype and the diagnosis of polysubstance dependence were removed in DSM-5. Either the binary DSM-IV diagnosis or the multinomial DSM-5 severity all have its utility in research. DSM-IV is still widely used by the research community.

The goal of this study was to translate the English version of the SSADDA into Chinese and examine the diag-

Complex Psychiatry 2020;6:62-67 
nostic reliability and validity of DSM-IV SD diagnoses in a Mandarin-speaking sample in Taiwan. Here, we report the findings on the diagnostic reliability and validity of the SSADDA-Chinese.

\section{Materials and Methods}

\section{Study Participants}

The study was conducted in the Chang Gung Memorial Hospitals (Kaohsiung and Keelung in Taiwan) and the Taipei City Psychiatric Center after being approved by the Institutional Review Boards at these facilities. All procedures performed in the study followed the ethical standards of each recruitment institution and the national research committee in Taiwan and the 1964 Declaration of Helsinki and its later amendments. Written informed consent was obtained from all study participants prior to them being interviewed, and the privacy rights of the study participants were observed throughout the study.

A total of 125 treatment-seeking patients with substance use problems were recruited from methadone clinics $(N=73,58.4 \%)$, psychiatric outpatient clinics $(N=18,14.4 \%)$, and therapy groups for substance-using adolescents $(N=34,27.2 \%)$ at 3 hospitals, where psychiatrists referred their patients to the study because they thought that they had substance use problems. The inclusion criteria were (a) age $\geq 12$ years, (b) having a substance use problem, and (c) being capable of reading Chinese and giving written informed consent.

The Chinese Semi-Structured Assessment for Drug Dependence and Alcoholism

The primary diagnostic instrument, SSADDA-Chinese, was derived from the English SSADDA [3]. A new section on ketamine use in English was developed by three psychiatrists (M.C.H., S.C.W., and H.K.) on the investigative team, as ketamine use disorder was not included in the original version of the SSADDA. After the ketamine section was finalized, two psychiatrists (C.K.C. and M.C.H.) and a research assistant in Taiwan and the co-investigator at Yale (B.Z.Y.), all fluent in both Chinese and English, translated the parent instrument into Chinese. The Chinese translation incorporated culturally relevant forms, names, and units for all substance categories. An independent bilingual translator backtranslated the initial draft from Chinese into English. The investigators who initiated the work (C.K.C., B.Z.Y., M.C.H., and S.C.W.) reviewed inconsistencies between the backtranslated and original English versions and amended the draft version through consensus to create the final instrument in Chinese.

\section{Structured Clinical Interview for DSM-IV, Clinician Version} (SCID)

We used a Chinese version of the SCID-IV [15], which has been validated and is widely used in clinical and research settings [1618 ], to test the concurrent validity of the SSADDA diagnoses. The SCID was designed for use by a clinician or mental health professional. In this study, the SCID interview was conducted by a trained psychiatrist and treated as a valid standard for psychiatric diagnosis. Lifetime diagnoses of SD and other psychiatric disorders were defined using the DSM-IV diagnostic criteria.
Table 1. Characteristics of the study sample and the DSM-IV life time diagnoses of substance dependence and psychatiric comorbidities $(N=125)$

\begin{tabular}{|c|c|c|}
\hline & $N$ or mean & $\%$ or $\mathrm{StD}$ \\
\hline \multicolumn{3}{|l|}{ Demographic data } \\
\hline Age, years & 36.9 & 13.7 \\
\hline \multicolumn{3}{|l|}{ Sex (male/female) } \\
\hline Male & 103 & 82.4 \\
\hline Female & 22 & 17.6 \\
\hline \multicolumn{3}{|l|}{ Education } \\
\hline Junior high school or lower & 60 & 48 \\
\hline Senior high school & 56 & 44.8 \\
\hline College or above & 9 & 7.2 \\
\hline \multicolumn{3}{|l|}{$S D$} \\
\hline Alcohol & 28 & 22.4 \\
\hline Ketamine & 13 & 10.4 \\
\hline Nicotine & 65 & 52 \\
\hline Methamphetamine & 39 & 31.2 \\
\hline Opioid & 78 & 62.4 \\
\hline Others & 4 & 3.2 \\
\hline \multicolumn{3}{|l|}{ Psychiatric comorbidities } \\
\hline Antisocial personality & 4 & 3.2 \\
\hline Depression & 29 & 23.2 \\
\hline Mania & 4 & 3.2 \\
\hline Psychosis & 1 & 0.8 \\
\hline ADHD & 4 & 3.2 \\
\hline Suicide attempt & 13 & 10.4 \\
\hline PTSD & 3 & 2.4 \\
\hline Generalized anxiety disorder & 1 & 0.8 \\
\hline OCD & 0 & 0 \\
\hline Social phobia & 4 & 3.2 \\
\hline Agoraphobia & 0 & 0 \\
\hline Panic disorder & 1 & 0.8 \\
\hline Pathological gambling & 12 & 9.6 \\
\hline
\end{tabular}

StD, standard deviation; SD, substance dependence; ADHD, attention deficit hyperactivity disorder; PTSD, posttraumatic stress disorder; OCD, obsessive-compulsive disorder.

\section{Study Procedure}

All interviews were conducted by research team members trained in the use of the SSADDA-Chinese. An interviewer-training course on the use of the SSADDA-Chinese was held from December 4 to 6, 2015, in Taipei. Y.Z.N., who has over 10 years of experience in the administration of the SSADDA, taught the course, and B.Z.Y. provided a side-by-side translation into Mandarin Chinese. After completing the training course, 4 psychiatrists and 2 research assistants on the project achieved criterion proficiency and were allowed to conduct in-person interviews of treatment-seeking patients in this study.

The order of interviews (the SSADDA-Chinese or the SCIDChinese first) was selected to ensure a comparable number of each order. We retested 31 participants within 8 weeks of the first test to evaluate the instrument's test-retest reliability.
Yang/Wang/Huang/Wang/Tsai/Huang/ Nuñez/Ng/Kranzler/Gelernter/Chen 
Table 2. Concurrent validity of the SSADDA-Chinese using the SCID-Chinese as a valid standard

\begin{tabular}{llll}
\hline DSM-IV diagnosis & DSM-IV diagnosis & \multicolumn{2}{l}{$\begin{array}{l}\text { Weighted kappa } \\
\text { coefficient }\end{array}$} \\
\cline { 3 - 4 } SSADDA & SCID & $N=125$ & $\begin{array}{l}N=99 \\
(\text { age } \geq 18)\end{array}$ \\
\hline Alcohol dependence & Alcohol dependence & 0.83 & 0.84 \\
Ketamine dependence & Ketamine dependence & 0.97 & 1 \\
Meth dependence & Meth dependence & 0.93 & 0.91 \\
OD & OD & 0.95 & 0.92 \\
Lifetime MDD & Past MDD & 0.7 & 0.72 \\
Lifetime MDD & Current MDD & 0.15 & 0.2 \\
Lifetime MDD & Lifetime MDD & 0.59 & 0.57 \\
\hline
\end{tabular}

SSADDA, Semi-Structured Assessment for Drug Dependence and Alcoholism; SCID, Structured Clinical Interview for DSM-IV; meth, methamphetamine; OD, opioid dependence; MDD, major depressive disorder.

Table 3. Test-retest reliability of the DSM-IV diagnosis using the SSADDA

\begin{tabular}{lll}
\hline $\begin{array}{l}\text { SSADDA test-retest DSM-IV } \\
\text { diagnosis }\end{array}$ & $\begin{array}{l}\text { Affected, } \\
n \text { (first, } \\
\text { second) test }\end{array}$ & $\begin{array}{l}\text { Weighted } \\
\text { kappa } \\
\text { coefficient }\end{array}$ \\
\hline $\mathrm{N}=31$ & & \\
Alcohol dependence & $(8,6)$ & 0.63 \\
Ketamine dependence & $(1,0)$ & 0.95 \\
Nicotine dependence & $(18,16)$ & 0.65 \\
Methamphetamine dependence & $(14,13)$ & 0.8 \\
OD & $(28,28)$ & 1 \\
MDD & $(8,10)$ & 0.84 \\
\hline
\end{tabular}

SSADDA, Semi-Structured Assessment for Drug Dependence and Alcoholism; OD, opioid dependence; MDD, major depressive disorder.

\section{Statistical Analyses}

To evaluate the validity of the diagnoses, we measured the concordance between diagnoses made using the Chinese versions of the SSADDA and the SCID for all 125 participants. We determined test-retest reliability in the subsample of patients interviewed twice with the SSADDA $(N=31)$. All statistical analyses were conducted using $\mathrm{R}$ (version 3.2.0), a free software environment for statistical computing [19]. Estimated concordance was evaluated using Cohen's $\kappa$ statistic for chance-corrected agreement [20]. Reliability and validity were categorized using suggested criteria: $<0.40$ as poor, $0.40-0.59$ as fair, $0.60-0.74$ as good, and $0.75-1.00$ as excellent [21].

Reliability and Validity of the Chinese SSADDA

\section{Results}

Most of the 125 participants were male $(n=103$, $82.4 \%)$. The mean age of all participants was 37.1 years (standard deviation $[\mathrm{StD}]=13.5)$ (Table 1$)$. Most participants (52.5\%) had a senior high school education, and $41.1 \%$ were married. A large majority $(86.3 \%)$ were nicotine dependent, $64.5 \%$ were opioid dependent, $42.7 \%$ were methamphetamine dependent, $15.3 \%$ were alcohol dependent, and $9.7 \%$ were ketamine dependent.

Based on concordance with the SCID-Chinese (Table 2), the SSADDA-Chinese showed good-to-excellent concurrent validity for dependence on alcohol $(\kappa=0.83)$, ketamine $(\kappa=0.97)$, methamphetamine $(\kappa=0.93)$, and opioids $(\kappa=0.95)$. We did not evaluate the concurrent validity of nicotine dependence, as it is not assessed in the SCID. Major depression was the most common non-substance-related diagnosis (Table 1). The SSADDA can be used to diagnose lifetime major depressive disorder (MDD), while the SCID differentiates depression into past MDD and current MDD, which we recoded into lifetime MDD. The concurrent validity of lifetime MDD by the SSADDA-Chinese using the SCID-Chinese diagnoses as a criterion measure was fair $(\kappa=0.59)$.

Because the study sample included subjects aged 1218 and age could affect diagnostic validity, we analyzed the participants who were aged $\geq 18$ years $(n=99$ or $80 \%)$ separately from those $<18$. The concurrent validity for all diagnoses for the 2 age groups separately was similar to that of the entire sample (Table 2).

The test-retest reliability (Table 3 ) of the SSADDAderived diagnoses of dependence on ketamine $(\kappa=0.95)$, methamphetamine $(\kappa=0.80)$, and opioids $(\kappa=1.00)$ was excellent, as was that for lifetime MDD $(\kappa=0.84)$. The test-retest reliability for dependence on alcohol $(\kappa=0.63)$ and nicotine $(\kappa=0.65)$ was good. Although we aimed to retest the 31 participants who agreed to be retested within 8 weeks of the first SSADDA interview, because of illness or incarceration (which was independent of their treatment), the test-retest interval was much longer than 8 weeks for 10 subjects, ranging from 261 to 590 days, with a mean of 407.9 days.

\section{Discussion}

This study examined the concurrent validity and testretest reliability of the Chinese version of the SSADDA for lifetime diagnoses of SD and MDD. The diagnosis of SD using the SSADDA-Chinese showed excellent con- 
current validity compared to the SCID-Chinese. The testretest reliability of SD diagnoses ranged from good to excellent.

Previous studies of the SSADDA have shown the instrument to be reliable in different population groups [3, $4,10,22]$. The current study also showed that the Chinese version of the SSADDA is reliable for assessing SD, but the small sample size for assessing the test-retest reliability and the fact that for some participants there was a delay in the retest interview due to illness or incarceration are limitations. Also, in the reliability study of the original SSADDA English version, besides the treatment-seeking patients, a set of 75 community respondents was included to gauge the utility in evaluating individuals of unknown diagnosis [3, 4]. It is a limitation in the current study for not including a set of community respondents.

Although the SSADDA is useful for a broad range of psychiatric traits in practice, the only non-substance-related psychiatric disorder that was prevalent enough for us to consider was lifetime MDD, of which test-retest reliability was excellent $(\kappa=0.84)$. However, the concurrent validity of the SSADDA was only fair when compared to the Chinese version of the SCID $(\kappa=0.59)$. The SSADDA identified 29 participants with lifetime MDD, while the SCID diagnosed only 14 participants with that diagnosis. The SSADDA was designed for the interviewee to answer most of the questions yes or no or to select from available designated options, so that the SSADDA requires less interpretation by the interviewer of the interviewee's responses. This difference may explain the discrepancies and reflects a limitation in using the SCID as a criterion measure for assessing validity.

Other limitations of this study include a small sample size and an assessment of dependence only on alcohol, ketamine, methamphetamine, and opioids. Because the prevalence of cocaine and cannabis use is comparatively low in Taiwan [12], users of these substances were not recruited to participate. Further, we could not evaluate the concurrent validity of nicotine dependence, as that diagnosis is not assessed by the SCID. We did not conduct an assessment of the interrater reliability of the instrument because it would have required additional interviewers to implement, and resources were limited.We only assessed the diagnostic concordance of the SSADDA-Chinese in relation to the SCID-Chinese. Other criterion measures (e.g., the MINI or clinical interview by a psychiatrist) could yield different findings. Additional types of validity (e.g., discriminant or construct validity) are required to support more fully the validity of the diagnoses obtained with the SSADDA-Chinese, but these were not assessed in the current study. Although the SSADDA can be used to make DSM-5 diagnoses, we used DSM-IV diagnoses in this study to allow a comparison with previous psychometric studies of the SSADDA $[3,4,10]$. Finally, the study sample was mostly male and approximately twothirds were opioid users, which limits the generalizability of the findings.

In summary, our data show good-to-excellent test-retest reliability and concurrent validity of the Chinese version of the SSADDA for the lifetime SD diagnoses. We conclude, therefore, that the SSADDA-Chinese is a reliable, valid, and practical tool for assessing SD diagnoses in Mandarin-speaking populations.

\section{Acknowledgements}

The authors express their deepest gratitude to Yi-Ting Chen, Si-Ming Wang, and Yi-Syuan Chen for helping with participant recruitment.

\section{Statement of Ethics}

All procedures performed in the study followed the ethical standards of each recruitment institution and the national research committee in Taiwan and the 1964 Declaration of Helsinki and its later amendments. Written informed consent was obtained from all study participants prior to them being interviewed, and the privacy rights of the study participants have always been observed.

\section{Conflict of Interest Statement}

Dr. Kranzler is a member of an advisory board for Dicerna Pharmaceuticals and of the American Society of Clinical Psychopharmacology's Alcohol Clinical Trials Initiative, which was sponsored in the past 3 years by AbbVie, Alkermes, Amygdala Neurosciences, Arbor Pharmaceuticals, Ethypharm, Indivior, Lilly, Lundbeck, Otsuka, and Pfizer. Drs. Kranzler and Gelernter are named as inventors on PCT patent application \#15/878640 entitled "Genotype-guided dosing of opioid agonists," filed on January 24,2018 . All other authors declared no conflicts of interest.

\section{Funding Sources}

This study was funded by the Chang Gung Medical Foundation (CMRPG8D0483, CGRPG2F0021, and CRRPG2G0051) in Taiwan. The funding sources had no involvement in the study design, collection, analysis, and interpretation of data, writing of the report, or the decision to submit the article for publication.
Yang/Wang/Huang/Wang/Tsai/Huang/ Nuñez/Ng/Kranzler/Gelernter/Chen 


\section{Author Contributions}

C.K. Chen, M.C. Huang, L.J. Wang, S.C. Wang, and B.Z. Yang participated in the design of the study and reviewed the literature. C.K.C., B.Z.Y., M.C.H.,. S.C.W., and H.K. created the SSADDAChinese version that included the development of a new ketamine section and translation into Chinese. Y. Nunez led the SSADDA training course and commented on the manuscript. L.J. Wang and B.Z. Yang drafted the manuscript. J. Gelernter commented on and critically reviewed the study design and article and supported the
SSADDA training and training course administratively and intellectually. L.J. Wang oversaw participant recruitment, and Y.C. Chen, Y.C. Huang, and M.C. Tsai recruited the patients. B.Z. Yang executed the statistical analysis, interpreted data, and drafted and revised the manuscript. Mei-H. Ng reviewed and revised the translation and provided input on issues related to substance use disorders in Taiwan. H.R. Kranzler commented on the study design and critically reviewed the manuscript for content and language usage. All authors read and approved the final manuscript.

\section{References}

1 United Nations Office on Drugs and Crime. Global overview of drug demand and supply: latest trends and cross-cutting issues. World Drug Report 2017. Vienna, Austria: United Nations; 2017.

2 Degenhardt L, Whiteford HA, Ferrari AJ, Baxter AJ, Charlson FJ, Hall WD, et al. Global burden of disease attributable to illicit drug use and dependence: findings from the Global Burden of Disease Study 2010. Lancet. 2013;382(9904):1564-74.

3 Pierucci-Lagha A, Gelernter J, Chan G, Arias A, Cubells JF, Farrer L, et al. Reliability of DSM-IV diagnostic criteria using the semistructured assessment for drug dependence and alcoholism (SSADDA). Drug Alcohol Depend. 2007;91(1):85-90.

4 Pierucci-Lagha A, Gelernter J, Feinn R, Cubells JF, Pearson D, Pollastri A, et al. Diagnostic reliability of the Semi-structured Assessment for Drug Dependence and Alcoholism (SSADDA). Drug Alcohol Depend. 2005; 80(3):303-12.

5 Bucholz KK, Cadoret R, Cloninger CR, Dinwiddie SH, Hesselbrock VM, Nurnberger JI Jr, et al. A new, semi-structured psychiatric interview for use in genetic linkage studies: a report on the reliability of the SSAGA. J Stud Alcohol. 1994;55(2):149-58.

6 Hesselbrock M, Easton C, Bucholz KK, Schuckit M, Hesselbrock V. A validity study of the SSAGA: a comparison with the SCAN Addiction. 1999;94(9):1361-70.

7 Denis CM, Gelernter J, Hart AB, Kranzler HR. Inter-observer reliability of DSM-5 substance use disorders. Drug Alcohol Depend. 2015;153:229-35.
8 Hogarth L, Martin L, Seedat S. Relationship between childhood abuse and substance misuse problems is mediated by substance use coping motives, in school attending South African adolescents. Drug Alcohol Depend. 2019;194:69-74.

9 Konkoly Thege B, Horwood L, Slater L, Tan MC, Hodgins DC, Wild TC. Relationship between interpersonal trauma exposure and addictive behaviors: a systematic review. BMC psychiatry. 2017;17(1):164.

10 Malison RT, Kalayasiri R, Sanichwankul K, Sughondhabirom A, Mutirangura A, Pittman $\mathrm{B}$, et al. Inter-rater reliability and concurrent validity of DSM-IV opioid dependence in a Hmong isolate using the Thai version of the Semi-Structured Assessment for Drug Dependence and Alcoholism (SSADDA). Addict Behav. 2011;36(1-2):156-60.

11 Quinn AE, Rosen RK, McGeary JE, Amoa F, Kranzler HR, Francazio S, et al. Translating the semi-structured assessment for drug dependence and alcoholism in the Western $\mathrm{Pa}$ cific: rationale, study design and reliability of alcohol dependence. Alcohol Alcohol. 2014; 49(5):525-30.

12 Chen WJ, Wu SC, Tsay WI, Chen YT, Hsiao $\mathrm{PC}, \mathrm{Yu} \mathrm{YH}$, et al. Differences in prevalence, socio-behavioral correlates, and psychosocial distress between club drug and hard drug use in Taiwan: results from the 2014 National Survey of Substance Use. Int J Drug Policy. 2017;48:99-107.

13 Huang K, Zhang L, Liu J. Drug problems in contemporary China: a profile of Chinese drug users in a metropolitan area. Int J Drug Policy. 2011;22(2):128-32.

14 Parkvall M. The world's 100 largest languages in 2007. Sweden: Nationalencyklopedin; 2010.
15 Phillips MR, Liu XH. Adapted Chinese version of Structured Clinical Interview for DSM-IV-TR Axis I Disorders, Research Version, Patient Edition (SCID-I/P) by Michael B. First, Robert L. Spitzer, Miriam Gibbon, and Janet B.W. Williams. Shanghai: Suicide Research and Prevention Center, Shanghai Mental Health Center; 2011.

16 Tseng MM, Fang D, Lee MB, Chie WC, Liu JP, Chen WJ. Two-phase survey of eating disorders in gifted dance and non-dance highschool students in Taiwan. Psychol Med. 2007;37(8):1085-96.

17 Liao SC, Chen WJ, Lee MB, Lung FW, Lai TJ, Liu CY, et al. Low prevalence of major depressive disorder in Taiwanese adults: possible explanations and implications. Psychol Med. 2012;42(6):1227-37.

18 Tsai CW, Gunnell D, Chou YH, Kuo CJ, Lee MB, Chen YY. Why do people choose charcoal burning as a method of suicide? An interview based study of survivors in Taiwan. J Affect Disord. 2011;131(1-3):402-7.

19 R Core Team. R: a language and environment for statistical computing and graphics. R Foundation for Statistical Computing; 2013.

20 Cohen J. Statistical power analysis for the behavioral sciences. 2nd ed. Hillsdale, New Jersey: Lawrence Erlbaum Associates; 1988.

21 Cicchetti DV, Sparrow SA. Developing criteria for establishing interrater reliability of specific items: applications to assessment of adaptive behavior. Am J Ment Defic. 1981; 86(2):127-37.

22 Feinn R, Gelernter J, Cubells JF, Farrer L, Kranzler HR. Sources of unreliability in the diagnosis of substance dependence. J Stud Alcohol Drugs. 2009;70(3):475-81. 